\title{
Polysomnography findings and risk factors for sleep-disordered breathing in patients with systemic sclerosis
}

\author{
Ebru Çakır Edis ${ }^{1}(\mathbb{D})$, Renginar Mutlucan Eraslan ${ }^{2}(\mathbb{D})$, Osman Hatipoğlu ${ }^{1}$ (i) \\ ${ }^{1}$ Department of Pulmonary Medicine, Trakya University Medical Faculty, Edirne, Turkey \\ ${ }^{2}$ Department of Pulmonary Medicine, Gönen State Hospital, Balikesir, Turkey
}

\begin{abstract}
Objectives: This study aims to evaluate polysomnography findings and risk factors for sleep-disordered breathing in patients with systemic sclerosis (SSc).

Patients and methods: Thirty-nine patients ( 2 males, 37 females; mean age: $51.1 \pm 12.5$ years; range, 21 to 76 years) who attended to the Rheumatology Clinic of Trakya University Medical Faculty between March 2014 and May 2014 were included in the study. Thoracic computed tomography, pulmonary function tests, carbon monoxide diffusion tests, echocardiography, and all-night polysomnography in a sleep laboratory were performed in all patients. Demographic and clinical characteristics of the patients were recorded. The Apnea-Hypopnea Index (AHI) scores were calculated. Disease activity was examined using the Medsger Disease Severity Scale (MDSS). Possible risk factors for sleep-disordered breathing were evaluated.

Results: Using the AHI of $\geq 5$ for obstructive sleep apnea (OSA), 21 (53.8\%) of the patients were classified as having OSA. There were no significant differences between the groups with and without OSA in terms of steroid use ( $p=0.4), C T$ involvement $(p=0.3)$, and Warrick ( $p=0.6)$ and MDSS scores $(p=0.5)$. Age, body mass index (BMI), and mean oxygen desaturation index were found to be significant in the univariate analysis $(p<0.1)$; however, the multivariate analysis revealed only BMI as significant risk factor of OSA $(p=0.028)$. In the multivariate analysis, the mean saturation was found to be significant risk factor for high pulmonary artery pressure $(p<0.001)$.
\end{abstract}

Conclusion: Although OSA is common in SSc, only increased BMI is a significant risk factor of OSA, but not lung involvement, Warrick scores, or MDSS scores.

Keywords: Medsger disease severity scale, obstructive sleep apnea, pulmonary hypertension, systemic sclerosis, Warrick scores.

Systemic sclerosis (SSc) is a multisystem autoimmune disease. The most common type of lung involvement in patients with SSc is interstitial lung disease (ILD), accounting for 90\% of all patients with SSc. ${ }^{1}$ Pulmonary hypertension is seen in 13 to $35 \%$ of patients with SSc. ${ }^{1}$ In addition, patients with SSc may present with airway disease and pleural involvement. Indirect pulmonary complications are frequently observed in patients with SSc, except for direct pulmonary involvement forms. These include gastroesophageal reflux, aspiration, infection, lung toxicity, malignancy, and respiratory muscle weakness related to the drugs used for the treatment. ${ }^{1}$

Sleep disorders are a major problem in SSc. The limited opening of the oral orifice in SSc patients, gastroesophageal reflux, and dysmotility play a role in the development of breathing disorders during sleep in these

Received: August 30, 2020 Accepted: November 03, 2020 Published online: January 20, 2021

Correspondence: Ebru Çakır Edis, MD. Trakya Üniversitesi Tıp Fakültesi Göğüs Hastalıkları Anabilim Dalı, 22030 Edirne, Türkiye. Tel: +90 284 - 2357641 / 1123 e-mail: ebruckr@yahoo.com

\section{Citation:}

Çakır Edis E, Mutlucan Eraslan R, Hatipoglu O. Polysomnography findings and risk factors for sleep-disordered breathing in patients with systemic sclerosis. Arch Rheumatol 2021;36(3):360-365. 
patients. ${ }^{2}$ In addition, it is considered that the systemic steroid which is used for the treatment of the disease may also lead to sleep problems. $^{3}$

The number of studies investigating sleep problems in patients with ILD has been increasing recently. ${ }^{4-6}$ However, there is only one study which investigated sleep problems with polysomnography (PSG) in SSc patients in the literature. ${ }^{2}$ In a recent retrospective study, overnight oximetry records were examined using electronic health records. ${ }^{7}$

In the present study, we aimed to evaluate PSG findings and risk factors for sleep-disordered breathing in patients with SSc.

\section{PATIENTS AND METHODS}

This prospective study was conducted at Trakya University Medical Faculty of Rheumatology Outpatient Clinic between March 2014 and May 2014. Patients older than 18 years who attended to our clinic and fulfilled the criteria of the American College of Rheumatology $(\mathrm{ACR})^{8}$ for $\mathrm{SSc}$ were included. Patients with heart failure, asthma, chronic obstructive pulmonary disease, kyphoscoliosis, Class IV dyspnea, connective tissue disease without SSc, predefined sleep disorders, and neurological or psychiatric disorders were excluded from the study. A total of 51 patients were screened and, among them, 40 patients consented for PSG. One patient with an advanced degree of kyphoscoliosis, which might have affected the statistical analysis, was excluded from the study. Finally, a total of 39 patients (2 males, 37 females; mean age: $51.1 \pm 12.5$ years; range, 21 to 76 years) were enrolled. A written informed consent was obtained from each patient. The study protocol was approved by the Trakya University Medical Faculty Ethics Committee (TÜTF-GOKAEK-2013-176). The study was conducted in accordance with the principles of the Declaration of Helsinki.

After their enrolment in the study, all the patients underwent a clinical evaluation, including a pulmonary function test (PFT), carbon monoxide diffusion test (DLCO), highresolution computed tomography (HRCT), and
PSG. Transthoracic echocardiography (TTE) was also performed in the cardiology laboratory.

After the clinical evaluation, the patients were scheduled for PSG in the sleep laboratory. All patients underwent an ear, nose and throat examination before the study. The patients were advised not to consume caffeinated beverages and alcohol and not to take medications affecting sleep architecture (e.g., antihistamines, antidepressants, or hypnotics). In all patients, the lengths and weights were measured, and body mass index (BMI) values were calculated (weight $[\mathrm{kg}] /$ lenght ${ }^{2}\left[\mathrm{~m}^{2}\right]$ ). Their symptoms in terms of breathing disorder during sleep (snoring, witnessed apneas, and excessive daytime sleepiness) were investigated, and all the patients completed the Epworth Sleepiness Scale (ESS). After consuming a light dinner, the patients attended to the laboratory at 09.00 PM. The study took place between 10.30 and 11.00 PM. When the patients woke up in the morning, the study was terminated.

All-night PSG (Technologies Astro-Med, West Warwick, RI, USA) was performed. The recording included electroencephalography (central and occipital), a two-channel electrooculogram, electromyogram, electrocardiogram, the oronasal flow (using a thermistor and a nasal pressure transducer), respiratory effort (using thoracoabdominal belt). Oxygen saturation was measured by pulse oximetry, snoring was detected by a microphone, and positions were recorded by means of a position censor.

The PSG findings were evaluated by $30-\mathrm{sec}$ windows following the recommended criteria of the American Academy of Sleep Medicine Guidelines (AASM) for Scoring of Sleep-2 Version 2012. Firstly, the sleep stages were scored. ${ }^{9}$ Sleep time, sleep efficiency, sleep latency, rapid eye movement (REM) latency, and wake after sleep onset (WASO) were recorded. Then, respiratory events were determined using the following criteria. $^{9}$

1. Apnea: Score a respiratory event as an apnea, when both of the following criteria are met:

a) There is a drop in the peak signal excursion by $\geq 90 \%$ of pre-event baseline

b) The duration of the $\geq 90 \%$ drop in sensor signal is $\geq 10 \mathrm{sec}$. 
Score an apnea as obstructive, if it meets apnea criteria and is associated with continued respiratory effort. The absence of respiratory effort is considered central sleep apnea.

2. Hypopnea: Score a respiratory event as a hypopnea if all of the following criteria are met:

a) The peak signal excursions drop by $\geq 30 \%$ of pre-event baseline

b) The duration of the $\geq 30 \%$ drop in signal excursion is $\geq 10 \mathrm{sec}$.

c) There is $\geq 3 \%$ oxygen desaturation.

3. Apnea-Hypopnea Index (AHI): It represents the number of total sleep apnea and hypopnea events per hour during sleep.

According to these criteria, obstructive, central and mixed apnea number, apnea index (AI), and AHI were evaluated for each patient. Saturation chases of the patients were performed by means of pulse oximeter; average and minimum oxygen percentages were evaluated separately according to awaken, asleep non-REM (NREM) and REM sleep stages. In addition, oxygen desaturation index (ODI) values were recorded.

After the recordings were evaluated, those called $\mathrm{AHI} \geq 5$ were regarded as obstructive sleep apnea (OSA). The OSA severity was regarded as mild, (5-15) moderate, (16-30) and severe (>30). Rapid eye movement-related OSA was accepted as $\mathrm{AHI} \geq 5$ and REM/NREM AHI $\geq 2 .{ }^{10}$

The HRCT was recorded by a 64-slice multidetector CT scan (Aquilion, Toshiba Medical Systems, USA) using standard references. The recordings were obtained during maximum inspiration and in the supine position. The patients' Warrick scores were calculated based on their HRCTs. ${ }^{11}$ The HRCT was calculated semiquantitatively as follows: $0=$ normal, $1=$ mild ( $<8$ points), $2=$ moderate (8-15 points), and $3=$ severe $(>15) \cdot{ }^{12}$

Standard respiratory function tests were applied to all patients using a standard computerized spirometry system (Sensor medics, $\mathrm{V}$ max 22 PFT SYSTEM, USA). A DLCO was performed and the actual DLCO level was corrected according to hemoglobin levels. The results were presented according to the expected values in terms of percentage.
All the patients underwent TTE (Vivid 7 Pro, GE, Horten, Norway, 2-4 $\mathrm{MHz}$ phased-array transducer) in the cardiology laboratory, and their systolic pulmonary artery pressures (sPAPs) were recorded.

Lung involvement was handled under a separate title within the Medsger Disease Severity Scale (MDSS), in which the disease activity of the scleroderma was assessed; and a grouping from 0 to 4 called as normal, mild, moderate, severe, and end stage was made. The grouping for 0 was evaluated as normal. In the disease which was assessed as normal at 0 , for the grouping of 1 (mild), the DLCO and force vital capacity (FVC) were $\geq 80 \%$, there was no fibrosis on radiography, and the sPAP was $<35 \mathrm{mmHg}$. In the grouping defined as 2 (moderate), the DLCO and FVC were between 70 and $79 \%$, there was fibrosis on radiography, there were bibasilar rales, and the sPAP was 35 to $49 \mathrm{mmHg}$. For the grouping defined as 3 (severe), the DLCO and FVC were between 50 and 69\%, and the sPAP was 50 to $64 \mathrm{mmHg}$. For the grouping defined as 4 (end stage), the DLCO and FVC were $<50 \%$, the sPAP was $>65 \mathrm{mmHg}$, and oxygen was needed. ${ }^{13}$

\section{Statistical analysis}

Statistical analysis was performed using the PASW version 17.0 software (SPSS Inc., Chicago, IL, USA). Continuous variables were expressed in mean \pm standard deviation or median (min-max), while categorical variables were expressed in number and percentage. In the univariate comparison, the Chi-square $\left(\chi^{2}\right)$ or $\mathrm{t}$-test was used, as appropriate. The correlation between the parameters was assessed using a non-parametric Spearman or Pearson correlation analysis. Variables with a $p$ value of $<0.1$ as a result of univariate analysis were included in the multivariate linear regression analysis. A $p$ value of $<0.05$ was considered statistically significant.

\section{RESULTS}

Thirty-nine patients were included in the study, 37 (95\%) of whom were female. The mean age was $51.1 \pm 12.5$. The mean BMI was $27.4 \pm 5.1 \mathrm{~kg} / \mathrm{m}^{2}$. Eleven (28\%) patients had a 
BMI of $>30 \mathrm{~kg} / \mathrm{m}^{2}$. In 14 (36\%) patients, SSc was classified as diffuse, and it was classified as limited in 25 (64\%) patients. The mean disease duration was $4.4 \pm 3.7$ years. Eight $(20.5 \%)$ of the patients were using steroids, six (15.4\%) of the patients were using azathioprine, 12 (30.7\%) of the patients were using methotrexate, and one $(2.6 \%)$ patient was taking mycophenolate. Twenty-nine (74\%) patients were non-smokers, five $(13 \%)$ were current smokers, and five (13\%) were ex-smokers.

When questioned about chest disease, the most frequently reported symptom was dyspnea ( $n=26,66.7 \%)$. Reflux symptoms were reported in $23(60.5 \%)$ patients, and dysphagia was reported in 12 (30.8\%) patients. Among the cardinal symptoms concerning respiratory disorder during sleep, snoring was experienced in nine (23.1\%) patients, excessive daytime sleepiness in $13(33.3 \%)$ patients, and witnessed apnea in two (5.1\%) patients. The mean ESS score was $4.5 \pm 6.1$.

When the HRCT results of the patients were investigated, 23 (59\%) had interstitial involvement. According to the Warrick score, three $(7.7 \%)$ of the patients were normal, and 13 (33.3\%) of them had mild lung involvement, seven (17.9\%) of them had moderate, and $16(41 \%)$ of them had severe involvement. The mean sPAP was $22.8 \pm 8.0 \mathrm{mmHg}$. In 10 patients, the sPAP was $>25 \mathrm{mmHg}$. The mean MDSS scores were calculated according to these data and were as normal in eight (20.5\%), mild in 12 (30.8\%),

Table 1. PSG findings of patients with SSc

\begin{tabular}{lc}
\hline PSG findings & Mean \pm SD \\
\hline Sleep duration (min) & $322.8 \pm 53.3$ \\
Sleep latency (min) & $14.8 \pm 11.6$ \\
REM latency (min) & $150.8 \pm 89.3$ \\
WASO (min) & $73.1 \pm 50.4$ \\
Sleep efficiency (\%) & $78.9 \pm 12.8$ \\
Sleep stages (\%) & \\
Stage 1 & $11.9 \pm 7.9$ \\
Stage 2 & $51.9 \pm 9.6$ \\
Stage 3 & $25.4 \pm 10.4$ \\
REM & $10.9 \pm 6.3$ \\
\hline PSG: Polysomnography; SSc: Systemic sclerosis; SD: Standard devia- \\
tion; REM: Rapid eye movement; WASO: Wake after sleep onset.
\end{tabular}

moderate in 11 (28.2\%), and severe in eight (20.5\%) patients.

The mean sleep duration was $322.8 \pm 53.3$ min. The mean sleep latency was $14.8 \pm 11.6 \mathrm{~min}$, and the mean REM latency was $150.8 \pm 89.3 \mathrm{~min}$. On average, the mean WASO was $73.0 \pm 50.4 \mathrm{~min}$ (Table 1).

In $12(30.7 \%)$ patients, a probe was not used to assess oxygen saturation due to fingertip fibrosis, and oxygen saturation was measured from different regions, such as the earlobe. The mean oxygen saturation during wakefulness was 94\%. During NREM and REM, it was 90\% and $92 \%$, respectively. During total sleep, it was $93 \%$. The minimum oxygen saturation was $84 \%$. The mean ODI was 4.96 .

When AHI $\geq 5$ was regarded for OSA, 21 (53.8\%) of the patients had OSA (Table 2). Five (12.8\%) had REM-dependent OSA.

The univariate analysis was performed to examine the effects of possible factors for OSA. Age, BMI, and mean ODI which were found to be significant in the univariate analysis were included in the multivariate analysis. It showed that only BMI was found to be significant risk factor of OSA $(p=0.028)$. However, there was no significant difference in terms of age, sex, complaints, physical examination, pulmonary function tests, severity of the disease, pulmonary hypertension, presence of $\mathrm{CT}$ involvement, or the extent of CT findings. After the current smoker and ex-smoker patients were removed from the analysis, 29 patients remained. Obstructive sleep apnea was detected in 16 (55.2\%) of these patients. However, the effect of smoking or not smoking on the frequency of OSA was not found to be significant. In addition, the mean saturation was found to be a significant risk factor of sPAP in the multivariate analysis $(\mathrm{p}<0.001)$.

Table 2. Severity of OSA according to the AHI

\begin{tabular}{lcc}
\hline OSA severity & $\mathrm{n}$ & $\%$ \\
\hline No OSA (AHI <5) & 18 & 46 \\
Mild OSA (AHI 5-15) & 16 & 41 \\
Moderate OSA (AHI 16-30) & 3 & 8 \\
Severe OSA (AHI >30) & 2 & 5 \\
\hline OSA: Obstructive sleep apnea; AHI: Apnea-Hypopnea Index. &
\end{tabular}


Based on the results of the study, continuous positive airway pressure (CPAP) was recommended for five patients who were symptomatic and with an AHI of $\geq 15$, and auto-CPAP was recommended for 10 patients who had the score of REM AHI $\geq 15$.

\section{DISCUSSION}

In the literature, there is only one study investigating sleep problems with PSG in SSc patients, which showed no respiratory disorder during sleep. ${ }^{2}$ In our study, the prevalence of OSA in patients with SSc was 53.8\%. There was no significant difference in terms of steroid use, lung involvement on HRCT, Warrick score, MDSS score, or pulmonary hypertension in those with and without OSA. In addition, OSA showed a significant correlation with BMI. There was also a correlation between the mean ODI and sPAP. Furthermore, the sleep duration, sleep quality, and REM ratio of the patients with SSc decreased, but they increased by Stage 1 and REM latency. In another study, the sleep efficiency and REM ratio decreased in patients with SSc. ${ }^{2}$

Based on an $\mathrm{AHI}$ of $\geq 5$, the prevalence of OSA in the general population is $24 \%$ for men and $9 \%$ for women. ${ }^{14}$ The OSA prevalence in patients with ILD ranges between 17 and $88 \%$, and most of these studies include patients with idiopathic pulmonary fibrosis. ${ }^{6,15}$ In a study which was conducted in Turkey investigating OSA in ILD, the overall prevalence was found to be $68 \%$, whereas this rate was $55.5 \%$ in SSc patients. ${ }^{4}$ In a study including PSG findings in patients with SSc, 27 patients were evaluated and none of the patients had $\mathrm{AHI} \geq 5$. $^{2}$ This difference may be the result of the methodology used to determine OSA. In another study by Prado et al., ${ }^{2}$ a pulse oximeter was not used, considering that it cannot measure sensitively due to fibrosis of the skin.

Conflicting results have been reported regarding the effect of $\mathrm{BMI}$ on sleeping parameters in the PSG studies in ILD patients. In a study conducted by Aydoğdu et al., ${ }^{5}$ there was a difference in terms of parameters regarding PSG between the two groups, one of them had a BMI of above $30 \mathrm{~kg} / \mathrm{m}^{2}$ and another one had a BMI of below $30 \mathrm{~kg} / \mathrm{m}^{2}$. In their studies, Pihtili et al. ${ }^{4}$ excluded patients who had
BMI $>30 \mathrm{~kg} / \mathrm{m}^{2}$, and found a weak correlation between the $\mathrm{AHI}$ and $\mathrm{BMI}$, consistent with our findings. However, in a study including patients with idiopathic pulmonary fibrosis, there was a strong correlation between the AHI and BMI. ${ }^{16}$

It has been thought that corticosteroid use may increase the risk for OSA by causing obesity. 3,17 On the other hand, Pihtili et al. ${ }^{4}$ found no significant difference between the groups with OSA and without OSA in terms of using steroid, which is consistent with our study.

To the best of our knowledge, there is no study in the literature investigating the relationship between involvement on HRCT and OSA in SSc patients. Pihtili et al. ${ }^{4}$ used chest X-rays to evaluate diffuse parenchyma involvement in ILD and found that the AHI was higher in patients with diffuse involvement.

There are few studies in the literature evaluating sleep quality in patients with SSc. ${ }^{18,19}$ Sariyildiz et al., ${ }^{19}$ evaluated sleep quality using the Pittsburg Sleep Quality Index (PSQI) in patients with SSc and found significantly higher scores for sleep quality, sleep latency, sleep disturbance, and total PSQI scores in the control group.

Nonetheless, there are some limitations to this study. One of the limitations is the lack of the control group in the study. Another limitation is that $\mathrm{SPAB}$ was evaluated using TTE, and right heart catheterization was unable to be performed.

In conclusion, our study results showed that about half of the SSc patients had OSA. The OSA frequency can be reduced by providing weight control. In addition, it may be beneficial to evaluate patients with hypoxia and no comorbidities other than SSc in terms of OSA and pulmonary hypertension. However, further largescale, randomized-controlled studies are needed to confirm these findings.

\section{Acknowledgment}

We thank Prof. Necdet Süt, MD for his valuable assistance for statistical analysis and Ömer N. Pamuk, MD from the rheumatology clinic for his helpful support.

\section{Declaration of conflicting interests}

The authors declared no conflicts of interest with respect to the authorship and/or publication of this article. 


\section{Funding}

This study received financial support from Scientific Research Projects (TÜBAP-2013-163).

\section{REFERENCES}

1. Solomon JJ, Olson AL, Fischer A, Bull T, Brown KK, Raghu G. Scleroderma lung disease. Eur Respir Rev 2013;22:6-19.

2. Prado GF, Allen RP, Trevisani VM, Toscano VG, Earley CJ. Sleep disruption in systemic sclerosis (scleroderma) patients: clinical and polysomnographic findings. Sleep Med 2002;3:341-5.

3. Valencia-Flores M, Resendiz M, Castaño VA, Santiago V, Campos RM, Sandino S, et al. Objective and subjective sleep disturbances in patients with systemic lupus erythematosus. Arthritis Rheum 1999;42:2189-93.

4. Pihtili A, Bingol Z, Kiyan E, Cuhadaroglu C, Issever $\mathrm{H}$, Gulbaran Z. Obstructive sleep apnea is common in patients with interstitial lung disease. Sleep Breath 2013;17:1281-8.

5. Aydoğdu M, Ciftçi B, Firat Güven S, Ulukavak Ciftçi T, Erdoğan Y. Assessment of sleep with polysomnography in patients with interstitial lung disease. Tuberk Toraks 2006;54:213-21.

6. Lancaster LH, Mason WR, Parnell JA, Rice TW, Loyd JE, Milstone AP, et al. Obstructive sleep apnea is common in idiopathic pulmonary fibrosis. Chest 2009;136:772-8.

7. Nokes BT, Raza HA, Cartin-Ceba R, Lyng PJ, Krahn LE, Wesselius L, et al. Individuals With Scleroderma May Have Increased Risk of Sleep-Disordered Breathing. J Clin Sleep Med 2019;15:1665-9.

8. Preliminary criteria for the classification of systemic sclerosis (scleroderma). Subcommittee for scleroderma criteria of the American Rheumatism Association Diagnostic and Therapeutic Criteria Committee. Arthritis Rheum 1980;23:581-90.

9. Berry RB, Brooks R, Gamaldo CE, Harding SM, Marcus CL, Vaughn BV. For the American Academy of Sleep Medicine. The AASM Manual for the Scoring of Sleep and As-sociated Evenets: Rules, Terminology and Tecnical Specifications, Version 2.0, Darien, IL: American Academy of Sleep Medicine; 2012.

10. Oksenberg A, Arons E, Nasser K, Vander T, Radwan H. REM-related obstructive sleep apnea: the effect of body position. J Clin Sleep Med 2010;6:343-8.

11. Warrick JH, Bhalla M, Schabel SI, Silver RM. High resolution computed tomography in early scleroderma lung disease. J Rheumatol 1991;18:1520-8.

12. Gutierrez M, Salaffi F, Carotti M, Tardella M, Pineda C, Bertolazzi C, et al. Utility of a simplified ultrasound assessment to assess interstitial pulmonary fibrosis in connective tissue disorders--preliminary results. Arthritis Res Ther 2011;13:R134.

13. Medsger TA Jr, Bombardieri S, Czirjak L, Scorza R, Della Rossa A, Bencivelli W. Assessment of disease severity and prognosis. Clin Exp Rheumatol 2003;21(3 Suppl 29):S42-6.

14. Young T, Palta M, Dempsey J, Skatrud J, Weber $\mathrm{S}$, Badr S. The occurrence of sleep-disordered breathing among middle-aged adults. N Engl J Med 1993;328:1230-5.

15. Turner GA, Lower EE, Corser BC, Gunther KL, Baughman RP. Sleep apnea in sarcoidosis. Sarcoidosis Vasc Diffuse Lung Dis 1997;14:61-4.

16. Mermigkis C, Chapman J, Golish J, Mermigkis D, Budur K, Kopanakis A, et al. Sleep-related breathing disorders in patients with idiopathic pulmonary fibrosis. Lung 2007;185:173-8.

17. Inönü Köseoğlu $H$, Kanbay A, Köktürk $O$. An important concomitancy: interstitial lung diseases and sleep related breathing disorders. Tuberk Toraks 2014;62:231-5.

18. Frech T, Hays RD, Maranian P, Clements PJ, Furst DE, Khanna D. Prevalence and correlates of sleep disturbance in systemic sclerosis--results from the UCLA scleroderma quality of life study. Rheumatology (Oxford) 2011;50:1280-7.

19. Sariyildiz MA, Batmaz I, Budulgan M, Bozkurt M, Yazmalar L, Inanir A, et al. Sleep quality in patients with systemic sclerosis: relationship between the clinical variables, depressive symptoms, functional status, and the quality of life. Rheumatol Int 2013;33:1973-9. 